\title{
Comparative Analysis of the Effects of Three Antithrombotic Regimens on Clinical Outcomes of Patients with Atrial Fibrillation and Recent Percutaneous Coronary Intervention with Stent. A Retrospective Cohort Study
}

\author{
Renato De Vecchis ${ }^{1, *}$, Cesare Baldi ${ }^{2}$ and Francesco Piemonte ${ }^{3}$ \\ ${ }^{1}$ Cardiology Unit, Presidio Sanitario Intermedio "Elena d'Aosta", ASL Napoli 1 Centro, Napoli, Italy \\ ${ }^{2}$ Heart Department, Interventional Cardiology, A.O.U. "San Giovanni di Dio e Ruggi D’Aragona”, Salerno, Italy \\ ${ }^{3}$ Cardiology Division, "San Giovanni di Dio" Hospital, ASL Napoli 2 Nord, Frattamaggiore (NA), Italy
}

\begin{abstract}
Introduction: Chronic atrial fibrillation (AF), coexisting with a history of recent coronary angioplasty with stent (PCI-S) represents an encoded indication for oral anticoagulation with warfarin (OAC) plus dual antiplatelet therapy (DAPT).

Methods: Using a retrospective cohort study we determined the respective impacts on cardio- vascular outcomes of three different pharmacologic regimens, i.e., triple therapy (TT) with warfarin + clopidogrel and aspirin, dual therapy (DT) with warfarin +clopidogrel or aspirin, dual antiplatelet therapy (DAPT) with clopidogrel + aspirin. Outcomes of interest were all-cause mortality, ischemic cardiac events, ischemic cerebral events, bleeding events. The inclusion criterion was the coexistence of an indication for OAC (e.g., chronic AF) with an indication for dual antiplatelet therapy due to recent PCl-S.

Results: Among the 98 patients enrolled, 48 (49\%), 31 (31.6\%), and 19(19.4\%) patients were prescribed TT, DT, and DAPT, respectively. Throughout a mean follow-up of $378 \pm 15.7$ days, there were no significant differences between the three regimens for all abovementioned outcomes. In particular, the total frequency of major bleeding was similar in the three groups: 5 cases $(10.4 \%)$ in TT, one case $(3.22 \%)$ in DT and no case in DAPT groups ( $p$ [chi-square test] $=0.1987$ ).

Conclusions: TT, DT and DAPT displayed similar efficacy and safety. Although the superiority of OAC vs. DAPT for stroke prevention in AF patients has been demonstrated by previous randomized trials, a smaller frequency of high thromboembolic risks' features in DAPT group of the present study may have prevented the observation of a higher incidence of ischemic stroke in this group.
\end{abstract}

Keywords: Atrial fibrillation, percutaneous coronary intervention, oral anticoagulant therapy, antithrombotic therapy, major adverse cardiovascular events, bleeding.

\section{INTRODUCTION}

A condition commonly found in clinical practice is the coexistence of chronic or persistent atrial fibrillation $(\mathrm{AF})$, which requires oral anticoagulation (OAC) [1] with angina pectoris or acute coronary syndrome (ACS), which requires percutaneous coronary intervention with stenting (PCI-S) [2], consistently followed by prolonged administration of dual antiplatelet therapy (DAPT) with acetylsalicylic acid (ASA) combined with another suitable platelet antiaggregant (e.g., a thienopyridine such as clopidogrel or prasugrel) [3]. The dual antiplatelet therapy is less effective than the OAC in the conditions where the latter is indicated (e.g., chronic atrial fibrillation) [1]. By contrast, the OAC, even when associated with ASA, is less effective than dual antiplatelet therapy to prevent intra-stent thrombosis or relapses of myocardial ischemia after PCl-S [2]. Thus,

*Address for correspondence: Dr. Renato De Vecchis, via P. Gaurico 21, 80125 Napoli, Italy; Tel: +39 3483313530; E-mail: devecchis.erre@virgilio.it, r.de.vecchis@alice.it the combination of OAC and dual antiplatelet therapy, a so- called "triple therapy", would seem to be the most rational solution for patients undergoing $\mathrm{PCl}-\mathrm{S}$ for acute coronary syndrome or stable effort angina in whom an indication coexists for long-term OAC [4-8]. However, this triple therapy (TT) with OAC, ASA, and clopidogrel is notoriously regarded to raise the opportunity for bleeding complications [8-9]. Thus, considering the very feared increase in bleeding inherent in the TT, experimental attempts have been made to reduce the risk of bleeding without diminishing the prophylactic effectiveness against AF-related cardioembolic events by adopting a simplified regimen, i.e., warfarin in combination with a sole antiplatelet agent (clopidogrel or ASA) $[8,10]$.

\footnotetext{
AIM

To make a comparative evaluation of the efficacy and safety endpoints of three different therapeutic strategies adopted within a population of patients, each of whom has a potential indication for both OAC and DAPT.
} 


\section{METHODS}

Monocentric case-records of outpatients with AF and history of previous $\mathrm{PCl}$ with implantation of one or more bare metal- or drug-eluting stents (either sirolimus or everolimus) were retrospectively examined. The time frame taken into account spans from January 2008 until to December 2013. Data concerning the patients included in our retrospective evaluation were taken from their electronic outpatient folders. Inclusion criterion was the documented indication for prophylaxis of thrombo-embolic events with warfarin (due to chronic or persistent atrial fibrillation, mechanical valve prosthesis, deep vein thrombosis) accompanied by the simultaneous indication in the same patient for prevention of a possible post-procedural intra-stent thrombosis by means of dual antiplatelet therapy (the latter being administered due to recent $\mathrm{PCl}-\mathrm{S}$ procedure for angina or acute coronary syndrome). Furthermore, for admission into the study, regular administration for an adequate period (not less than 1 month) should have been documented, for one of the following drug regimens: (a) triple therapy (TT) with warfarin plus the combination therapy with ASA and clopidogrel; (b) dual therapy (DT) with warfarin plus either ASA or clopidogrel (i.e., one only of the two above mentioned antiplatelet agents, or (c) dual antiplatelet therapy (DAPT) with ASA and clopidogrel. Furthermore, in the case where $A F$ had been the indication for OAC, patients in whom AF had begun before the coronary stent implantation were considered separately from patients in whom AF had occurred after the stent implantation. Because of the retrospective nature of our study, the investigators did not interfere in the selection of patients assigned to the three treatment regimens. The pharmacologic associations and their doses were determined for each patient, according to the full discretion of the treating physicians and based on criteria that included the patient's general condition, comorbidities, and especially the profiles of both thrombotic and bleeding risk according to the $\mathrm{CHA}_{2} \mathrm{DS}_{2}$-VASc [11] and HAS-BLED [11] scores, respectively. (See explanatory synopses for both the scores shown in Tables 1 and 2). The data were meticulously collated and elaborated to build the summarizing tables.

\section{Statistical Analysis}

All statistical tests were made with a commercially available statistical analysis program (SPSS 15.0 for Windows, SPSS Inc., Chicago, IL, USA). The distribution of the data was assessed using the D'Agostino-Pearson test. Continuous variables displaying normal distribution were expressed as mean $\pm \mathrm{SD}$, while values with asymmetric distribution were expressed as medians with interquartile ranges. Categorical variables were presented as percentages (\%). The comparisons were made by means of the one-way analysis of variance (ANOVA) (continuous variables) or by applying the chi-square test (categorical variables). The impact of the three antithrombotic therapies on several outcomes, such as major adverse cardiovascular events (MACEs), total bleeding, major bleeding events, and total events (MACEs plus bleeding) was evaluated with the KaplanMeier method, and differences between treatment

Table 1: $\mathrm{CHA}_{2} \mathrm{DS}_{2}$-VASc Score for Estimating the Risk of Stroke in Patients with Atrial Fibrillation

\begin{tabular}{|c|c|c|}
\hline & Condition & Points \\
\hline \hline C & Congestive heart failure (or left ventricular systolic dysfunction) & 1 \\
\hline H & Hypertension: blood pressure consistently above 140/90 mmHg (or treated hypertension on medication) & 1 \\
\hline$A_{2}$ & Age $\geq 75$ years & 2 \\
\hline D & Diabetes mellitus & 2 \\
\hline$S_{2}$ & Vascular disease (e.g. peripheral artery disease, myocardial infarction, aortic plaque) & 1 \\
\hline V & Age 65-74 years & 1 \\
\hline A & Sex category (i.e. female sex) & 1 \\
\hline SC & & \\
\hline
\end{tabular}

The $\mathrm{CHA}_{2} \mathrm{DS}_{2}$-VASc score is a refinement of $\mathrm{CHADS}_{2}$ score and extends the latter by including additional common stroke risk factors, such as vascular disease, age 65-74 years and sex category (i.e. female sex). The maximum $\mathrm{CHA}_{2} \mathrm{DS}_{2}$-VASc score is 9 (for age, either the patient is $\geq 75$ years and gets two points, is between 65-74 and gets one point, or is under 65 and does not get points). Note that female gender only scores one point if the patient has at least one other risk factor, and does not score any points in isolation.

Legenda: TIA, transient ischemic attack. 
Table 2: HAS-BLED Score for Assessing the Bleeding Risk During Oral Anti-Coagulation among Patients with AF

\begin{tabular}{|c|c|c|}
\hline & Clinical feature & Points \\
\hline \hline H & Hypertension (systolic blood pressure $>160 \mathrm{mmHg}$ ) & 1 \\
\hline $\mathrm{A}$ & $\begin{array}{c}\text { Abnormal renal function (defined as the presence of chronic dialysis or renal transplantation or } \\
\text { serum creatinine } \geq 200 \mu \mathrm{mol} / \mathrm{L} \text { [> 2.3 mg/dL]) }\end{array}$ & 1 \\
\hline & $\begin{array}{c}\text { Abnormal liver function (defined as chronic hepatic disease (eg. cirrhosis) or biochemical } \\
\text { evidence of significant hepatic derangement (eg. bilirubin > 2x upper limit of normal, in } \\
\text { association with AST/ALT/ALP >3x upper limit normal) }\end{array}$ & 1 \\
\hline S & Stroke (previous history of stroke) & 1 \\
\hline B & Bleeding (Major bleeding history [anemia or predisposition to bleeding]) & 1 \\
\hline L & Labile INRs (refers to unstable/high INRs or poor time in therapeutic range [eg<60\%]) & 1 \\
\hline E & Elderly (age $\geq 65$ y) & 1 \\
\hline D & Drug therapy (concomitant therapy such as antiplatelet agents, NSAID's, steroids) & 1 \\
\hline & Alcohol intake (consuming 8 or more alcoholic drinks per week) & Maximum score: 9 points \\
\hline
\end{tabular}

Risk of major bleeding: score $0=1 \% /$ year, score $5=12.5 \% /$ year.

HAS-BLED score interpretation A score of 3 or more indicates an increased risk of bleeding that would be sufficient to justify the prudence or more frequent assessment. Physicians should also remember that the risk of bleeding may be changed and the 'HAS-BLED score can help you understand what correct: for example, discontinuation of therapy with aspirin and a better blood pressure control may be two ways to reduce the risk of bleeding.

Legenda: AST, aspartate aminotransferase; ALT, alanine aminotransferase; ALP, alkaline phosphatase; INR, International Normalized Ratio; NSAIDs, non-steroidal anti-inflammatory drugs.

groups were estimated with the log-rank test. Ethical approval was not requested, since it is usually not prescribed for retrospective cohort studies in Italy because patients cannot be identified.

\section{RESULTS}

98 patients were retrospectively recruited. Their main baseline characteristics are reported in Table 3 . Among them, 48 patients (48.9\%) were prescribed TT, $31(31.6 \%)$ received DT and $19(19.4 \%)$ were prescribed DAPT. Mean $\mathrm{CHADS}_{2}, \mathrm{CHA}_{2} \mathrm{DS}_{2}-\mathrm{VASc}$, and HAS-BLED scores were 1.8 $\pm 1.5,5.3 \pm 1.6$, and $2.3 \pm 0.5$, respectively. After a mean follow-up of $378 \pm$ 15 days, the absolute rates of MACEs, total bleeding, major bleeding, and total events (MACE plus bleeding) were $21.4 \%, 18.4 \%, 7.1 \%$, and $39.8 \%$, respectively. In particular, no significant differences across the three therapy groups were identified for both MACEs and total bleedings (Table 4, Figures 1-3). Likewise, no differences were found in regards to the site of major or minor bleeding across the three therapy categories (Table 5), except for only genitourinary hemorrhages (a kind of minor bleeding) that exhibited a significant difference in distribution among the three groups $(0 / 48$ cases in TT patients, $1 / 31$ cases among DT patients and $3 / 19$ cases among DAPT patients; $p$ [chi -square test]=0.013).Moreover, the lack of significant differences was noticeable in the rate of hemorrhagic relapses by comparing the three therapy groups (Table 5). Likewise, by using the Kaplan-Meier analysis, no significant differences were observed in the survival free from total MACEs and total bleeding events across the three groups (Figures 4 and $\mathbf{5}$ ).

In our experience, the TT duration never exceeded the limit of three months. Therefore, in our caserecords there were a number of patients for whom dual therapy (DT) was planned as a therapeutic starting strategy (for atrial fibrillation or other conditions entailing thromboembolic risk among patients also requiring simultaneous prophylaxis of intra- stent thrombosis) together with another group of patients, where DT with warfarin plus an antiplatelet drug (ASA or clopidogrel) represented the continuation of the original TT scheme. The therapy was either stopped after one month if a bare metal stent (BMS) had been implanted or was interrupted after a variable period (extending from one to three months) if a drug-eluting stent (DES) had been used. In this scenario, AF cases that occurred after stent implantation (i.e., 20 patients) were significantly less frequent compared to those in which AF occurrence had preceded the percutaneous coronary procedure (i.e., 78 patients) ( $p$ [chi square test] $<0.0001$ ). However, although the risk of bleeding might appear more pronounced in the cases of AF that had arisen after PCl-S because of the higher expected number of DES implants in this subset and because of the related need for prolonged dual antiplatelet therapy, 
Table 3: Baseline Characteristics

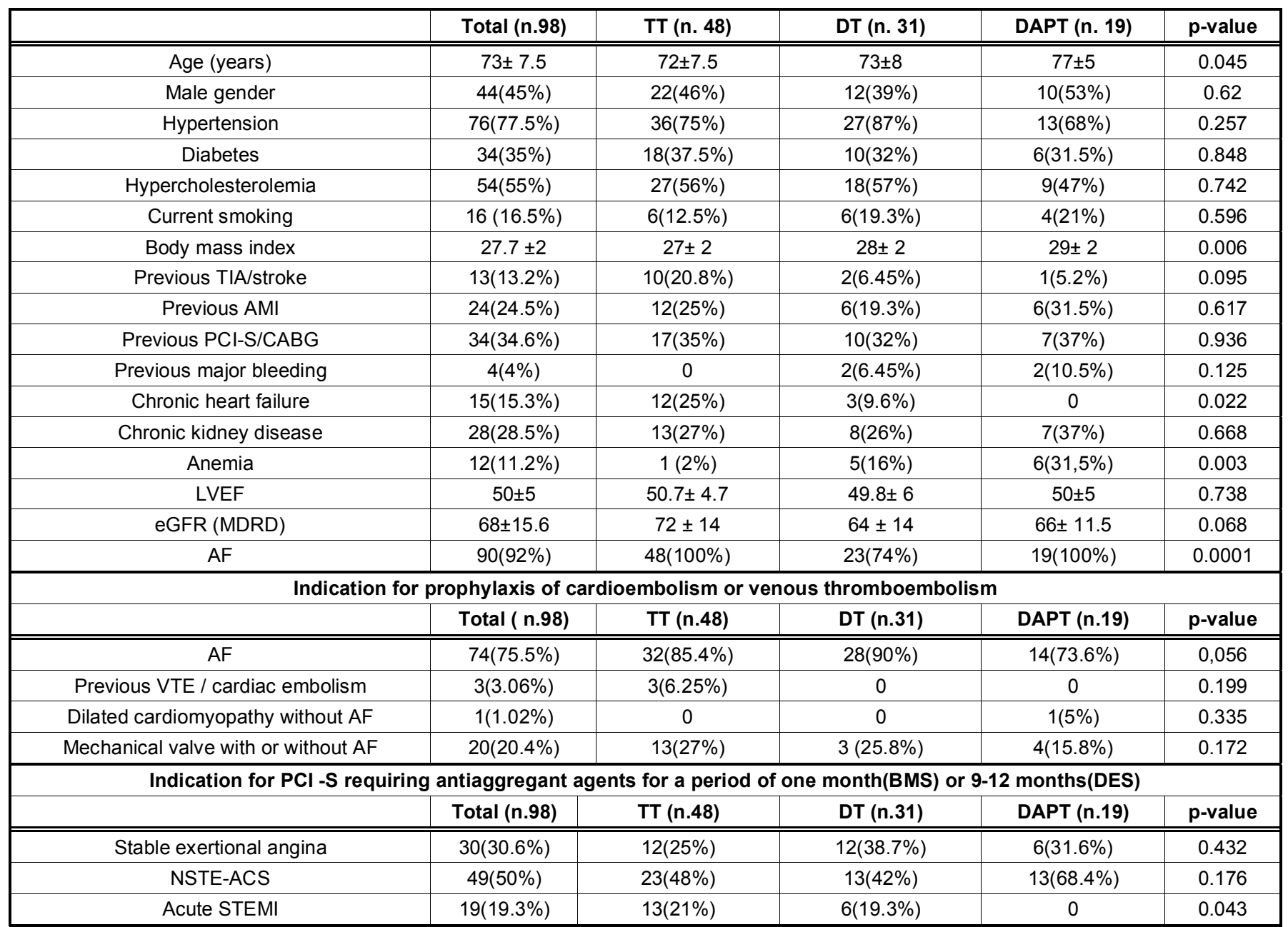

Data are reported as absolute number (percentage) or mean \pm standard deviation. TT= triple therapy (warfarin +acetylsalicylic acid and clopidogrel); DT= dual therapy (warfarin + acetylsalicylic acid or clopidogrel); DAPT= dual antiplatelet therapy (acetylsalicylic acid + clopidogrel); TIA= transient ischemic attack; AMI= acute myocardial infarction; $\mathrm{PCl}-\mathrm{S}=$ percutaneous coronary intervention with stent; $\mathrm{CABG}=$ coronary artery bypass graft; LVEF= left ventricular ejection fraction; eGFR= estimated glomerular filtration rate; MDRD: Modification of Diet in Renal Disease Study equation; $A F=$ atrial fibrillation; VTE= venous thromboembolism; BMS= bare metal stent; DES= drug eluting stent; NSTE-ACS= non-STelevation acute coronary syndrome; STEMI=ST-elevation myocardial infarction.

Table 4: Incidence of Adverse Events According to Therapeutic Strategy at Discharge, after a Follow-Up of One Year

\begin{tabular}{|c|c|c|c|c|c|}
\hline & Total $(n=98)$ & TT $(n=48)$ & DT $(n=31)$ & DAPT $(n=19)$ & $p$-value \\
\hline Total MACE & $21(21.43 \%)$ & $13(27.1 \%)$ & $4(12.9 \%)$ & $4(21 \%)$ & 0.324 \\
\hline Total ACS & $5(5.1 \%)$ & $2(4.2 \%)$ & $1(3.22 \%)$ & $2(10.5 \%)$ & 0.480 \\
\hline Unstable angina & $4(4.08 \%)$ & $1(2 \%)$ & $1(3.22 \%)$ & $2(10.5 \%)$ & 0.277 \\
\hline Stent thrombosis & $1(1.02 \%)$ & $1(2 \%)$ & 0 & 0 & 0.591 \\
\hline DVT/PE & $3(3.06)$ & $3(6.25 \%)$ & 0 & 0 & 0.199 \\
\hline Stroke/TIA & $1(1.02 \%)$ & $1(2 \%)$ & 0 & 0 & 0.591 \\
\hline Total bleeding & $18(18.4 \%)$ & $8(16.66 \%)$ & $6(19.35 \%)$ & $4(21 \%)$ & 0.903 \\
\hline Major & $7(7.1 \%)$ & $4(8.3 \%)$ & $2(6.45 \%)$ & $1(5.3 \%)$ & 0.893 \\
\hline
\end{tabular}

Data are reported as absolute number (percentage). TT= triple therapy (warfarin +acetylsalicylic acid and clopidogrel); DT= dual therapy (warfarin +acetylsalicylic acid or clopidogrel); DAPT= dual antiplatelet therapy (acetylsalicylic acid + clopidogrel); MACE= major adverse cardiovascular events; ACS= acute coronary syndromes; $\mathrm{AMI}=$ acute myocardial infarction; $\mathrm{TIA}=$ transient ischemic attack; DVT= deep vein thrombosis; $\mathrm{PE}=$ pulmonary embolism. 


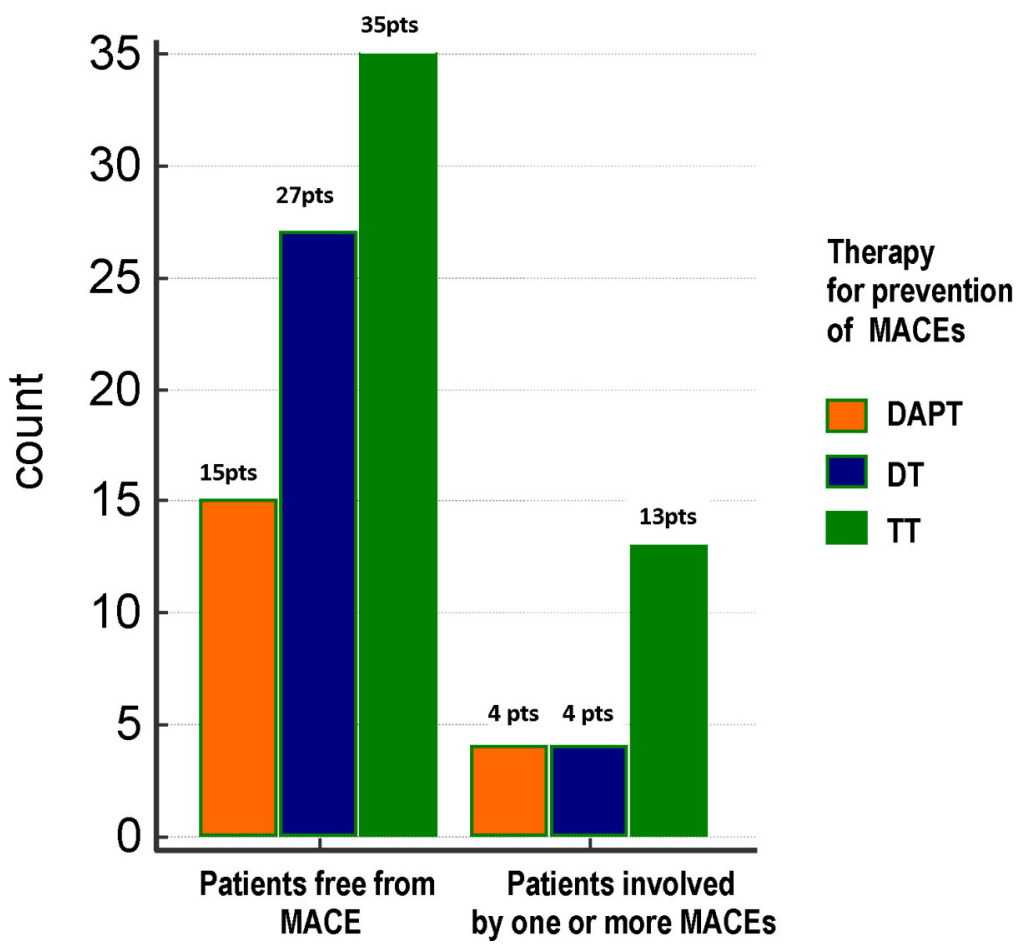

Figure 1: Distribution of total MACEs across the three treatment groups. No significant differences were found between groups as regards the frequency of total MACEs $(21 \%, 12.9 \%$ and $27.1 \%$ in DAPT,DT and TT groups, respectively; $p$ [chi-square test $]=0.324$.

Legenda: TT, triple therapy (warfarin +acetylsalicylic acid[ASA] and clopidogrel); DT, dual therapy (warfarin +ASA or clopidogrel); DAPT, dual antiplatelet therapy (ASA + clopidogrel); MACE, majoradverse cardiovascular events; pts, patients.

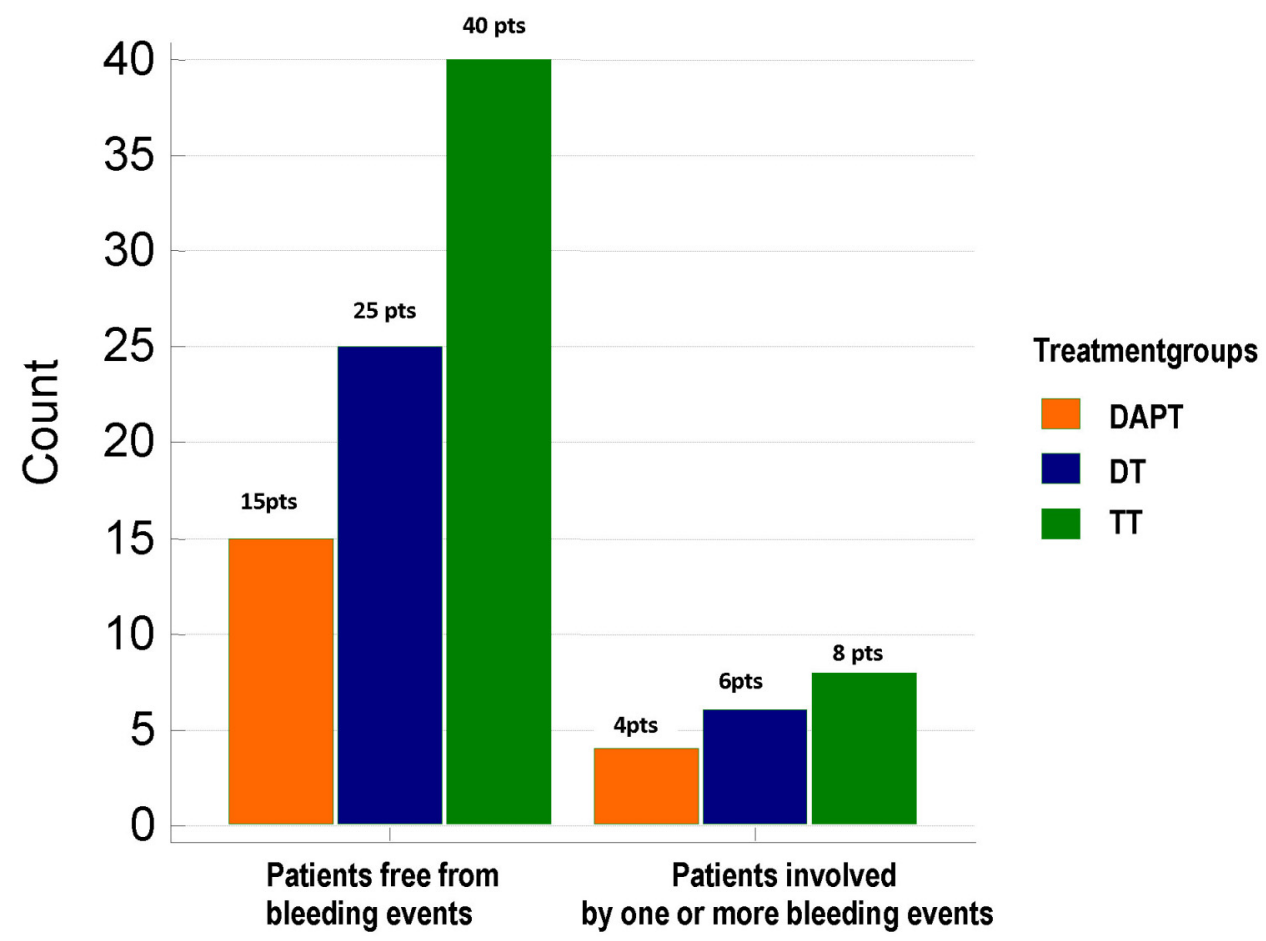

Figure 2: Distribution of total bleeding events across the three treatment groups. No significant differences were found between groups as regards the frequency of total bleeding events $(21 \%, 19.35 \%$ and $16.6 \%$ in DAPT, DT and TT groups, respectively; $p[$ chi-square test $]=0.903$.

Legenda: TT, triple therapy (warfarin +acetylsalicylic acid[ASA] and clopidogrel); DT, dual therapy (warfarin +ASA or clopidogrel); DAPT, dual antiplatelet therapy (ASA + clopidogrel); pts, patients. 


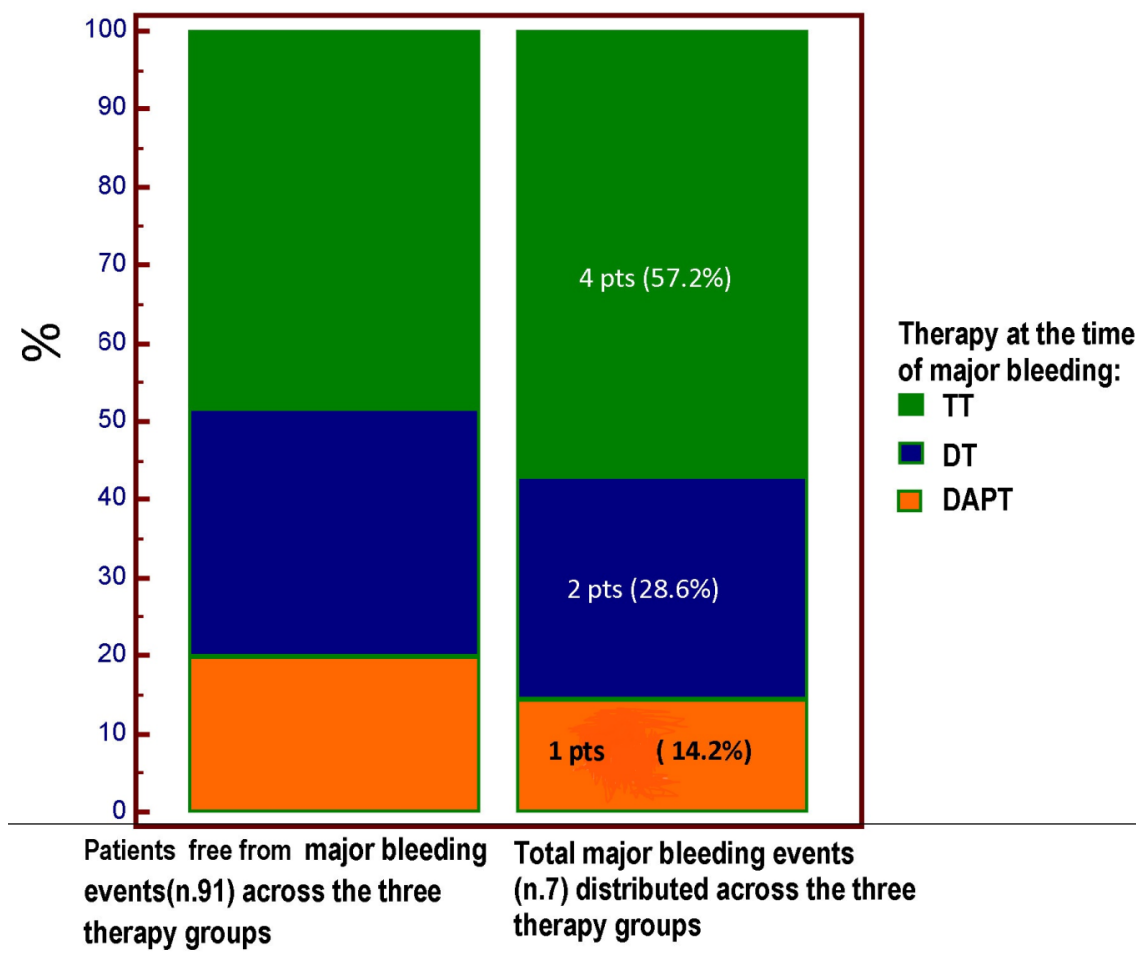

Figure 3: The type of antithrombotic regimen at the time of major bleeding is highlighted. Four out of seven major bleeding events occurred in the course of TT regimen, however no significant difference ( $p$ [chi squared test] $=0.893$ ) was demonstrated for the incidence of major bleeding across the three treatment groups, among whom the TT group was the most numerous (48 pts vs. 31 pts in the DT group and only 19 patients in the DAPT group).

Legenda: TT, triple therapy (warfarin +acetylsalicylic acid[ASA] and clopidogrel); DT, dual therapy (warfarin +ASA or clopidogrel); DAPT, dual antiplatelet therapy (ASA + clopidogrel); pts, patients.

Table 5: Sites of Bleeding Found during One Year Follow-Up

\begin{tabular}{|c|c|c|c|c|c|}
\hline & $\begin{array}{l}\text { Total n. of pts } \\
\quad(\mathrm{n} .98)\end{array}$ & TT (n.48) & DT (n.31) & DAPT (n.19) & $p$-value \\
\hline $\begin{array}{l}\text { Bleeding events } \\
\text { (major and minor) }\end{array}$ & $18(18.4 \%)$ & $8(16.66 \%)$ & $6(19.35 \%)$ & $4(21 \%)$ & 0.903 \\
\hline \multicolumn{6}{|c|}{ Major bleeding events } \\
\hline total & $7(7.1 \%)$ & $4(8.3 \%)$ & $2(6.45 \%)$ & $1(5.2 \%)$ & 0.893 \\
\hline gastrointestinal & $2(2.04 \%)$ & $1(2.1 \%)$ & $1(3.2 \%)$ & 0 & 0.735 \\
\hline genitourinary & $2(2.04 \%)$ & $1(2.1 \%)$ & 0 & $1(5.2 \%)$ & 0.442 \\
\hline $\begin{array}{l}\text { other (iliopsoas } \\
\text { haematoma) }\end{array}$ & $1(1.02 \%)$ & $1(2.1 \%)$ & 0 & 0 & 0.591 \\
\hline \multicolumn{6}{|c|}{ Minor bleeding events } \\
\hline genitourinary & $4(4.1 \%)$ & 0 & $1(3.2 \%)$ & $3(15.8 \%)$ & 0.013 \\
\hline \multicolumn{6}{|c|}{ Relapses of minor bleeding } \\
\hline $\begin{array}{l}\text { Minor bleeding events }>1 \\
\text { in the same patient }\end{array}$ & $8(8.16 \%)$ & $5(10.4)$ & $2(6.45 \%)$ & $1(5.26 \%)$ & 0.719 \\
\hline
\end{tabular}

Data are reported as number (percentage). pts= patients; TT= triple therapy (warfarin+ acid acetylsalicylic acid and clopidogrel); DT=dual therapy (warfarin + acid acetylsalicylic or clopidogrel); DAPT=dual antiplatelet therapy (acetylsalicylic acid + clopidogrel). 


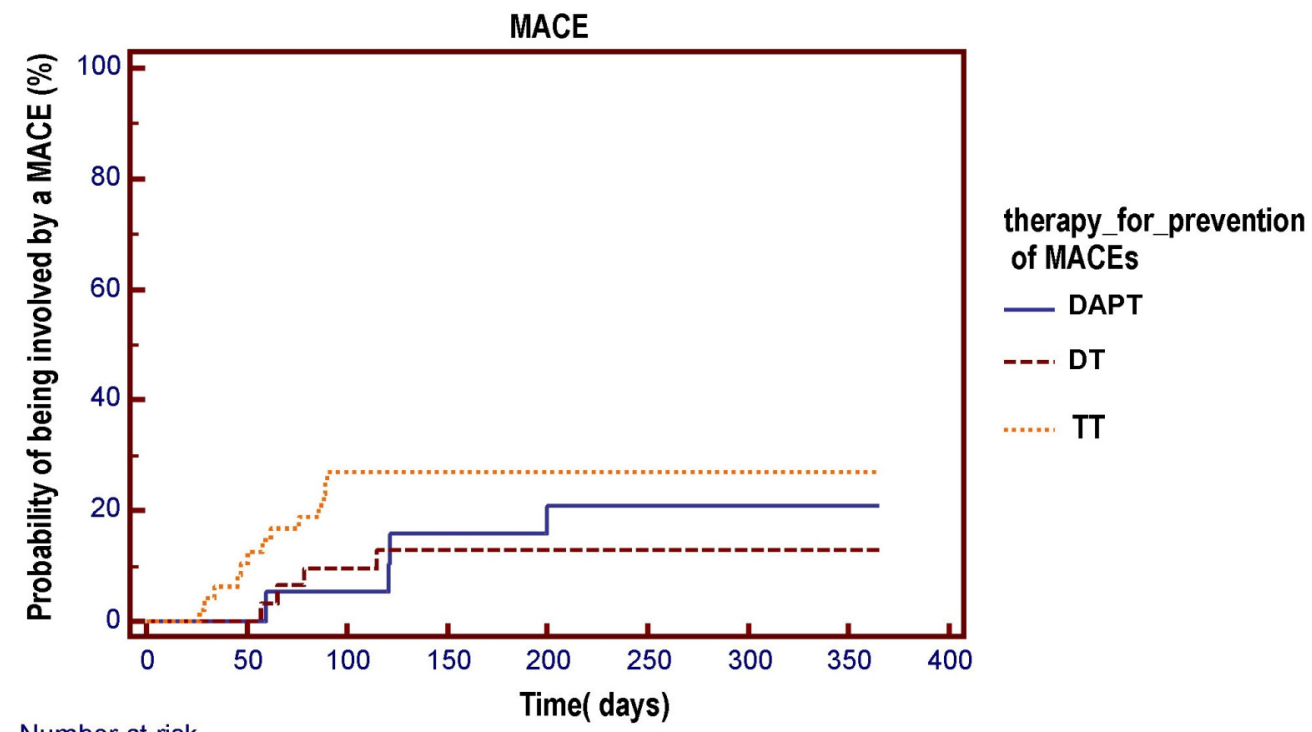

$\begin{array}{ccccccccc}\begin{array}{l}\text { Number at risk } \\ \text { Group: DAPT } \\ 19\end{array} & 19 & 18 & 16 & 15 & 15 & 15 & 15 & 0 \\ \text { Group: DT } & & & & & & & & \\ 31 & 31 & 28 & 27 & 27 & 27 & 27 & 27 & 0 \\ \text { Group: TT } & & & & & & & & \\ 48 & 42 & 35 & 35 & 35 & 35 & 35 & 35 & 0\end{array}$

Figure 4: The Kaplan-Meier curve is used to compare the respective probabilities of being involved by a MACE across the three antithrombotic treatment groups. No significant differences across the three pharmacologic regimens were observed in the survival free from total MACE over a one year follow-up. $p$ (log-rank test $)=0.284$.

Legenda: DAPT, dual antiplatelet therapy (acetylsalicylic acid $[\mathrm{ASA}]+$ clopidogrel); DT= dual therapy (warfarin +ASA or clopidogrel); TT, triple therapy (warfarin +ASA and clopidogrel).

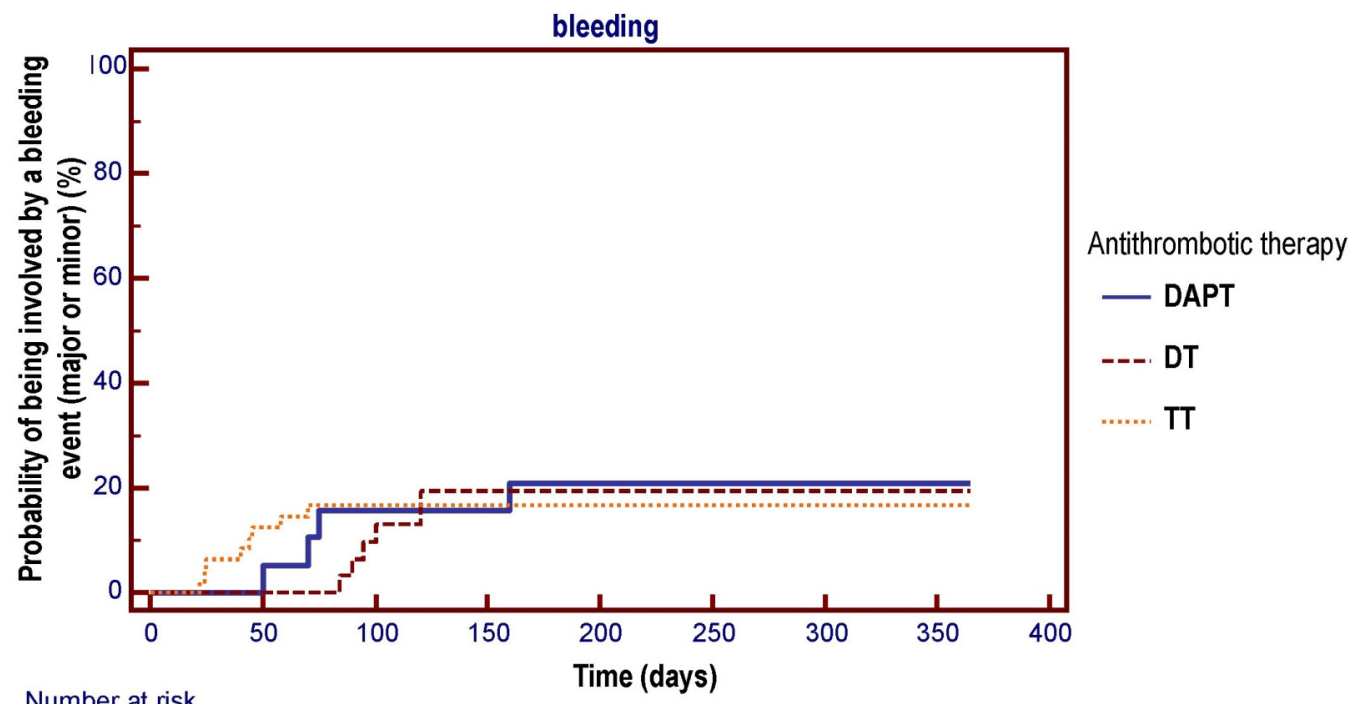

Number at risk

\begin{tabular}{|c|c|c|c|c|c|c|c|c|}
\hline \multicolumn{9}{|l|}{ Group:DAPT } \\
\hline 19 & 18 & 16 & 16 & 15 & 15 & 15 & 15 & 0 \\
\hline \multicolumn{9}{|l|}{ Group: DT } \\
\hline 31 & 31 & 27 & 25 & 25 & 25 & 25 & 25 & 0 \\
\hline \multicolumn{9}{|l|}{ Group:Tт } \\
\hline 48 & 42 & 40 & 40 & 40 & 40 & 40 & 40 & 0 \\
\hline
\end{tabular}

Figure 5: The Kaplan-Meier curve is used to compare the respective probabilities of being involved by one or more bleeding events (major or minor) across the three antithrombotic treatment groups. No significant differences across the three pharmacologic regimens were observed in the survival free from total bleeding events over a one year follow-up. $p$ (log-rank test) $=0.955$.

Legenda: DAPT, dual antiplatelet therapy (acetylsalicylic acid [ASA] + clopidogrel); DT= dual therapy (warfarin +ASA or clopidogrel); TT, triple therapy (warfarin +ASA and clopidogrel). 
Table 6: Frequency of the Bleeding Events within the Two AF Subsets Enrolled in the Study: AF Pre-Existing with Respect to PCI-S (78 Patients) or Arisen after PCI-S (20 Patients)

\begin{tabular}{|l|c|c|c|}
\hline & $\begin{array}{c}\text { AF already present } \\
\text { before the interventional procedure } \\
\text { of coronary stenting (no.78patients) }\end{array}$ & $\begin{array}{c}\text { AF arisen } \\
\text { during the year following the interventional } \\
\text { procedure of coronary stenting (no.20 patients) }\end{array}$ & $\begin{array}{c}p \text {-value } \\
\text { (chi square test) }\end{array}$ \\
\hline \hline Bleeding events & 14 & 4 & 0.9106 \\
\hline
\end{tabular}

Legenda: $\mathrm{AF}$, atrial fibrillation; $\mathrm{PCI}$-S, percutaneous coronary intervention with stent implantation.

the incidence of bleeding events in the two subsets was comparable $(p=0.9116)$ (see Table 6).

Moreover, importantly, all the 4 major bleeding events reported among patients assigned to TT occurred while administering the triple drug combination. Actually, in regards to the two major bleeding events signaled in the DT group, they involved patients who had been assigned from the first moment to DT, due to their HAS-BLED score that indicated they were at high risk of bleeding. Indeed, bleeding did not occur in cases when DT was administered as a continuation of the original TT after the latter had been stopped. In fact, no case of major bleeding was found in the timeframe subsequent to the switch from TT to DT.

\section{DISCUSSION}

The present analysis focused on outpatients, each of whom had experienced a condition requiring oral anticoagulation (OAC) with warfarin (AF, deep venous thrombosis, history of ascertained or suspected cardioembolism, or mechanical cardiac prosthesis). This need for OAC was complicated by the concomitant need for antiaggregant agents, since every patient had recently undergone a PTCA with stent implantation, thus requiring a dual antiplatelet regimen, according to the current guidelines [2-3]. Therefore, two different scenarios were represented in our study, depending on whether the condition requiring $O A C$ had preceded the stenting procedure or had taken place after the PCl-S.In the first case, (i.e., AF preceding the $\mathrm{PCl}$ ), the adoption of $\mathrm{BMS}$ was consistently preferred on occasion of the subsequent angioplasty with stenting, considering that this type of coronary prosthesis doesn't cause a sustained loss of endothelium, thus requiring a relatively short duration (usually, one month) of the dual antiplatelet therapy (ASA + thienopyridine) for the prophylaxis of intra-stent thrombosis. This is very important because with this method the combined regimen of an anticoagulant joined with a dual antiplatelet treatment is unlikely to generate a high amount of bleeding events, given the relatively short duration (not more than one month) of the dual antiplatelet therapy as scheduled by this protocol. Instead, in the second case, the AF occurs after the patient has already undergone a PCl-S that(in high income countries) generally entails the use of a drug-eluting stent (DES),i.e., a stent that elutes an immunosuppressant drug. In this setting, very challenging problems arise because in the case of DES the prophylaxis of the intra-stent thrombosis with dual antiplatelet therapy should be conducted for at least 12 months [12-13], which amplifies the risk that a possible combination regimen comprising the OAC can result in bleeding complications. This concern is the reason for the current trend to maintain TT for a relatively short period (1-3 months in our experience) and then convert it into a less demanding regimen, namely, a hybrid protocol that provides for a single antiplatelet agent (e.g., ASA or clopidogrel) in addition to warfarin or, alternatively, the maintenance of only dual antiplatelet therapy. As presented in the Results,the three antithrombotic regimens of TT, DT, and DAPT that we used in our study exhibited comparable efficacy and safety throughout the prescribed period of follow-up (mean duration among the 98 patients retrospectively examined $=378 \pm 15.7$ days). The absolute one-year rates of major bleeding with TT, DT, and DAPT were $8.3 \%, 6.45 \%$, and $5.2 \%$, respectively ( $p$ [chi-square test $]=0.893)$. By comparing the three different therapeutic regimens tested in our study (TT, DT and DAPT), no significant difference was detected regarding the incidence of MACEs (acute coronary syndromes, need for percutaneous or surgical revascularization, or ischemic stroke) or all-cause mortality. In regards to this aspect, the literature data are somewhat contradictory. In fact, based on the results of the ACTIVE-W study [1], there is documentation of higher efficacy of oral warfarin compared to combination therapy clopidogrel + ASA for the prevention of the ischemic stroke in AF patients. In the above mentioned study, the clopidogrel plus ASA group showed significantly more MACEs, such as stroke, non-central nervous system systemic embolism, myocardial infarction, or vascular death compared to those who received oral warfarin $(p=0.0003)$. Thus, the study was stopped early owing to the clear superiority of the warfarin therapy. By contrast, there are several studies in the literature that demonstrate a substantially 
comparable preventive efficacy against MACEs exerted by the three abovementioned regimens $[8,14]$. Moreover, recent data from the prospective, randomized WOEST study [15] have even shown superior efficacy of DT (with warfarin plus clopidogrel) over TT in regards to the occurrence of MACEs. In addition, in their recent study concerning the prospective, multicenter warfarin and coronary stenting (WAR-STENT) registry, conducted among patients with chronic atrial fibrillation and recent coronary angioplasty with stenting (mostly using bare metal stents), Rubboli et al. [16] detected no evidence of superiority of the TT regimen compared to the simplified scheme consisting of a single antiplatelet agent (ASA or clopidogrel) added to an oral anticoagulant (warfarin). Indeed, in this study, no differences in terms of antithrombotic efficacy were evident, based on the comparison between TT, DT (i.e., warfarin supplemented by either ASA or clopidogrel) and DAPT with ASA and clopidogrel. Furthermore, in the above mentioned study, even an alleged and greatly feared high incidence of major bleeding was not proven in the group of patients assigned to TT scheme. At 12 months, in fact, the frequency of major bleeding events was 4\% (14/339 patients), $5 \%$ (1/20 patients) and 2\% (1/42 patients) in the TT, DT and DAPT groups, respectively.

In patients requiring both the $O A C$ and the prophylaxis of intra-stent thrombosis, our study did not show a superiority of TT regimen compared to alternative schemes consisting of dual antiplatelet therapy or combined administration of warfarin with only one antiplatelet agent with regard to efficacy outcomes (MACEs and mortality from all causes) as well as for the safety endpoints (bleeding events). Therefore, our study adds to the already quite numerous series [8,14-16] of preexisting investigations that have disavowed the alleged higher anti-ischemic protection through the practice of adding two, rather than one, antiplatelet agent on top of OAC in patients with atrial fibrillation and recent PCI-S. Really, not even the recent official guidelines provide convincing evidence that triple therapy has better therapeutic efficacy in this clinical setting, considering that European and American position documents categorize recommendations for triple therapy (TT) as "Level of Evidence C" [17-18].

\section{STUDY LIMITATIONS}

The small sample size and its retrospective observational design are the main limitations of the study. Thus, possible indication biases are likely to have occurred, for example, due to the allocation to the TT group of patients presenting the most severe clinical picture and the highest $\mathrm{CHA}_{2} \mathrm{DS}_{2}$-VASc score. In this way, due to the lack of balance of clinical characteristics across the three groups, the possible higher antithrombotic efficacy of triple therapy may have been hidden or undersized. Moreover, in the present study, the significance of the anti-ischemic protective effect and the potential risk for bleeding of other drug combinations (prasugrel + ASA, ticagrelor + ASA) were not explored, because the data harvested from our outpatient population dates back a few years ago when these novel antiplatelet agents were not used in a routine manner at our Institute, whilst the new oral anticoagulants, alternative to warfarin (dabigatran, rivaroxiban, apixaban, etc.) [19] had not been validated yet for clinical use.

\section{CONCLUSIONS}

Based on our retrospective study, the three antithrombotic regimens TT, DT, and DAPT showed comparable efficacy and safety. Further studies with larger sample sizes, preferably modeled as randomized clinical trials, should be planned for comparing the various feasible regimens (with anticoagulant plus single or dual antiplatelet therapy or consisting of the association of two platelet antiaggregants) among patients with indication for OAC and the concomitant need for protection against stent thrombosis due to recent $\mathrm{PCI}-\mathrm{S}$.

\section{DECLARATION}

The authors of this article declare that they have no conflicts of interest concerning the contents of their paper.

\section{REFERENCES}

[1] Connolly S, Pogue J, Hart R, Pfeffer M, Hohnloser S, Chrolavicius S, Pfeffer M, Hohnloser S, Yusuf S, for the for the ACTIVE Writing Group of the ACTIVE Investigators. Clopidogrel plus aspirin versus oral anticoagulation for atrial fibrillation in the Atrial Fibrillation Clopidogrel Trial With Irbesartan for Prevention of Vascular Events (ACTIVEW): a randomised controlled trial. Lancet 2006; 367: 1903-12. http://dx.doi.org/10.1016/S0140-6736(06)68845-4

[2] Steg PG, James SK, Atar D, Badano LP, BlömstromLundqvist $C$, Borger MA, et al. ESC Guidelines for the management of acute myocardial infarction in patients presenting with ST-segment elevation: the Task Force on the Management of ST-Segment Elevation Acute Myocardial Infarction of the European Society of Cardiology (ESC). Eur Heart J 2012; 33: 2569-619. http://dx.doi.org/10.1093/eurheartj/ehs215

[3] Yusuf S, Zhao F, Mehta SR, Chrolavicius S, Tognoni G, Fox KK. Clopidogrel in Unstable Angina to Prevent Recurrent 
Events Trial Investigators. Effects of clopidogrel in addition to aspirin in patients with acute coronary syndromes without ST-segment elevation. N Engl J Med 2001; 345(7): 494-502. http://dx.doi.org/10.1056/NEJMoa010746

Orford JL, Fasseas P, Melby S, Burger K, Steinhubl SR, Holmes DR, Berger PB. Safety and efficacy of aspirin, clopidogrel, and warfarin after coronary stent placement in patients with an indication for anticoagulation. Am Heart $J$ 2004; 147(3): 463-7. http://dx.doi.org/10.1016/j.ahj.2003.06.004

[5] Rubboli A, Brancaleoni R, Colletta M, Herzfeld J, Sangiorgio $\mathrm{P}$, Di Pasquale G. Contemporary antithrombotic treatment after coronary stenting in patients with indication for longterm anticoagulation. Minerva Cardioangiol 2006; 54(5): 68793.

[6] Hermosillo AJ, Spinler SA. Aspirin, clopidogrel, and warfarin: is the combination appropriate and effective or inappropriate and too dangerous? Ann Pharmacother 2008; 42(6): 790805. http://dx.doi.org/10.1345/aph.1K591

Rubboli A, Verheugt FW. Antithrombotic treatment for patients on oral anticoagulation undergoing coronary stenting: a review of the available evidence and practical suggestions for the clinician. Int J Cardiol 2008; 123(3): 2349.

http://dx.doi.org/10.1016/j.ijcard.2007.02.017

[8] Lamberts $M$, Gislason $G H$, Olesen JB, Kristensen SL, Schjerning Olsen AM, Mikkelsen A, Christensen CB, Lip GY, Køber L, Torp-Pedersen C, Hansen ML. Oral anticoagulation and antiplatelets in atrial fibrillation patients after myocardial infarction and coronary intervention. J Am Coll Cardiol 2013; 62(11): 981-9. http://dx.doi.org/10.1016/j.jacc.2013.05.029

Lamberts M, Olesen JB, Ruwald MH, Hansen CM, Karasoy D, Kristensen SL, Køber L, Torp-Pedersen C, Gislason GH, Hansen ML. Bleeding after initiation of multiple antithrombotic drugs, including triple therapy, in atrial fibrillation patients following myocardial infarction and coronary intervention: a nationwide cohort study. Circulation 2012; 126(10): 1185-93.

http://dx.doi.org/10.1161/CIRCULATIONAHA.112.114967

[10] Rubboli A, Milandri M, Castelvetri C, Cosmi B. Meta-analysis of trials comparing oral anticoagulation and aspirin versus dual antiplatelet therapy after coronary stenting. Clues for the management of patients with an indication for long-term anticoagulation undergoing coronary stenting. Cardiology 2005; 104(2): 101-6. http://dx.doi.org/10.1159/000086918

[11] Lane DA, Lip GY. Use of the CHA2DS2-VASc and HASBLED scores to aid decision making for thromboprophylaxis in nonvalvular atrial fibrillation. Circulation 2012; 126(7): 860http://dx.doi.org/10.1161/CIRCULATIONAHA.111.060061
[12] Silber S, Hoffmeister HM, Bode C. [Optimal platelet inhibition after coronary stent implantation. Current status]. [Article in German] Herz 2008; 33(4): 244-53. http://dx.doi.org/10.1007/s00059-008-3138-9

[13] Park SJ, Kang SM, Park DW. Dual antiplatelet therapy after drug-eluting stents: defining the proper duration. Coron Artery Dis 2014; 25(1): 83-9.

http://dx.doi.org/10.1097/MCA.0000000000000066

[14] Rubboli A, Schlitt A, Kiviniemi T, Biancari F, Karjalainen PP, Valencia J, Laine M, Kirchhof P, Niemelä M, Vikman S, Lip GY, Airaksinen KE, AFCAS Study Group. One-year outcome of patients with atrial fibrillation undergoing coronary artery stenting: an analysis of the AFCAS registry. Clin Cardiol 2014; 37(6): 357-64. http://dx.doi.org/10.1002/clc.22254

[15] Dewilde WJ, Oirbans T, Verheugt FW, Kelder JC, De Smet BJ, Herrman JP, Adriaenssens T, Vrolix M, Heestermans AA, Vis MM, Tijsen JG, van 't Hof AW, tenBerg JM, WOEST study investigators. Use of clopidogrel with or without aspirin in patients taking oral anticoagulant therapy and undergoing percutaneous coronary intervention: an open-label, randomised, controlled trial. Lancet 2013; 381(9872): 110715.

http://dx.doi.org/10.1016/S0140-6736(12)62177-1

[16] Rubboli A, Saia F, Sciahbasi A, Bacchi-Reggiani ML, Steffanon L, Briguori C, Calabrò P, Palmieri C, Rizzi A, Imperadore $F$, Sangiorgi $G M$, Valgimigli $M$, Carosio $G$, Steffenino G, Galvani M, Di Pasquale G, La Vecchia L, Maggioni AP, Bolognese L, WARfarin and Coronary STENTing (WAR-STENT) Study Group. Outcome of patients on oral anticoagulation undergoing coronary artery stenting: data from discharge to 12 months in the Warfarin and Coronary Stenting (WAR-STENT) Registry. J Invasive Cardiol 2014; 26(11): 563-9.

[17] Lip GY, Huber K, Andreotti F, Arnesen H, Airaksinen KJ, Cuisset $\mathrm{T}$, Kirchhof $\mathrm{P}$, Marín F; European Society of Cardiology Working Group on Thrombosis. Management of antithrombotic therapy in atrial fibrillation patients presenting with acute coronary syndrome and/or undergoing percutaneous coronary intervention/ stenting. Thromb Haemost 2010; 103(1): 13-28.

\section{http://dx.doi.org/10.1160/TH09-08-0580}

[18] Faxon DP, Eikelboom JW, Berger PB, Holmes DR, Bhatt DL, Moliterno DJ, Becker RC, Angiolillo DJ. Consensus document: antithrombotic therapy in patients with atrial fibrillation undergoing coronary stenting. A North-American perspective. Thromb Haemost 2011; 106(4): 572-84. http://dx.doi.org/10.1160/TH11-04-0262

[19] Ahmad Y, Lip GY, Apostolakis S. New oral anticoagulants for stroke prevention in atrial fibrillation: impact of gender, heart failure, diabetes mellitus and paroxysmal atrial fibrillation. Expert Rev Cardiovasc Ther 2012; 10(12): 1471-80. http://dx.doi.org/10.1586/erc.12.148

Received on 12-06-2015

(c) 2015 De Vecchis et al.; Licensee Lifescience Global.

This is an open access article licensed under the terms of the Creative Commons Attribution Non-Commercial License (http://creativecommons.org/licenses/by-nc/3.0/) which permits unrestricted, non-commercial use, distribution and reproduction in any medium, provided the work is properly cited. 